\title{
Teor de CLA em leites produzidos em diferentes regiões do Estado do Rio Grande do Sul
}

\section{CLA content in milk produced in different regions of Rio Grande do Sul}

\author{
Gitane Fuke, ${ }^{*}$ José Laerte Nörnberg, ${ }^{* *}$ Isadora Lencina Rodrigues, ${ }^{* *}$ Ana Paula Binato de Souza, ${ }^{* *}$ Mariana Ercolani Novack, ${ }^{* *}$ \\ Aline Sobreira Bezerra**
}

\begin{abstract}
Resumo
Diversos fatores afetam a qualidade do leite dentro de uma propriedade leiteira, muitas alterações são responsáveis pelas variações da qualidade nutricional do leite. Portanto, objetivou-se determinar o teor de ácidos graxos e o de ácido linoleico conjugado, em leites produzidos em diferentes regiões do Rio Grande do Sul. Amostras de leite foram obtidas em três diferentes laticínios do Rio Grande do Sul, situados nas regiões do Noroeste, Norte e Sul, produzidas nas diferentes regiões e estações climáticas, do ano de 2010 e que são comercializadas na cidade de Santa Maria/RS. O perfil de ácidos graxos e de ácido linoleico conjugado foi determinado por cromatografia gasosa. O principal ácido graxo insaturado foi o oleico (18:1n9c). O teor de ômega-3 foi maior no inverno $(0,75 \%)$ e o teor de ômega- 6 presente no leite foi maior na estação do verão para as regiões Norte e Noroeste. A razão $\omega-6 / \omega-3$ variou de 3:1 a 5:1 com relação às médias observadas neste estudo. Os níveis de ácido linoleico conjugado no leite aumentaram nas estações da primavera e do inverno, com média de 10,13 mg. O conteúdo CLA de leites produzidos na região Sul do Brasil possuem valores superiores aos relatados para os produtos lácteos de países em climas temperados.
\end{abstract}

Palavras-chave: ácido linoleico conjugado, ácidos graxos, leite.

\begin{abstract}
Several factors affect the quality of milk in a dairy property, many changes are responsible for variçãoes the nutritional quality of milk. Therefore, the objective was to determine the content of fatty acids and conjugated linoleic acid in milk produced in different regions of Rio Grande do Sul Milk samples were obtained in three different dairy Rio Grande do Sul, in the regions of the Northwest North and South, produced in different regions and seasons of the year 2010 and are marketed in the city of Santa Maria / RS. The profile of fatty acids and conjugated linoleic acid content was determined by gas chromatography. The major unsaturated fatty acid was oleic $(18: 1 \mathrm{n} 9 \mathrm{c})$. The omega-3 content was higher in winter $(0.75 \%)$ and omega- 6 content in milk was higher in the summer season for the North and Northwest. The reason $\omega-6 / \omega-3$ ranged from 3:1 to 5:1 with respect to the means observed in this study. The levels of conjugated linoleic acid in milk increased in the spring and winter, with an average of $10.13 \mathrm{mg}$. The CLA content of milk produced in southern Brazil have values higher than those reported for dairy products from countries in temperate climates.
\end{abstract}

Keywords: conjugated linoleic acid, fatty acids, milk.

\section{Introdução}

A demanda por leite com propriedades funcionais decorrentes do ácido linoleico conjugado (CLA) está em plena expansão em países desenvolvidos, de forma que muitos pesquisadores em todo o mundo têm avaliado diferentes formas de aumentar o teor de CLA no leite de ruminantes (Rodríguez-Alcala e Fontecha, 2007). O CLA representa uma mistura de isômeros posicionais e geométricos do ácido linoleico (cis-9, cis-12 octadecadienoico), com duplas ligações conjugadas, sendo encontrado em vários produtos alimentícios, em maiores proporções nos lácteos, carnes bovinas, e em quantidades menores na suína, aves e óleo vegetal (Hur et al., 2007). O CLA tem sido relatado por apresentar efeitos benéficos na saúde, relacionado com doenças e utilizando modelos animais e culturas de células derivadas de humanos e animais. Assim, o CLA tem demonstrado efeitos benéficos à saúde como anticarcinogênico (Kelly at al., 2007), redução na deposição de gordura corporal, redução no desenvolvimento de aterosclerose (Serra et al., 2009), estimulação da função imune (Bhattacharya et al., 2006) e redução da glicose sanguínea (Belury et al., 2002). Portanto, a determinação do CLA em leites, que são uma das principais fontes naturais de CLA, é uma questão relevante para a saúde.

O teor médio no leite pode variar de 0,30 a 0,55g por $100 \mathrm{~g}$ de ácidos graxos (Dhiman et al., 1999). Parodi (2003), em pesquisa sobre a composição de ácidos graxos, em especial o CLA, observou valores de 2 a $37 \mathrm{mg}$ de lipídio/100g. A variabilidade de valores é principalmente a fatores ambientais e regionais, e as dietas das vacas, que parece ser o principal determinante da variação da composição de ácidos graxos no leite, assim com as práticas agrícolas, fatores genéticos e ambientais influenciam

\footnotetext{
* Universidade Federal de Santa Maria (UFSM) - Centro de Educação Superior Norte (CESNORS); Departamento Ciências da Saúde, Palmeira das Missões, Rio Grande do Sul, Brasil. Autor para correspondência. Email: gifuke@yahoo.com.br

**Universidade Federal de Santa Maria (UFSM) - Departamento Ciência e Tecnologia de Alimentos; Santa Maria, Rio Grande do Sul, Brasil.
} 
nestes valores (Collomb et al., 2002; Ledoux et al., 2005; Eifert et al., 2006; Collomb et al., 2006). Portanto, a composição de CLA em alimentos lácteos industrializados disponíveis para consumo pode apresentar diferenças entre regiões e entre os tipos de produtos lácteos.

O Rio Grande do Sul (RS) apresenta diversidade edafoclimática e condições propícias para o desenvolvimento da pecuária leiteira com animais de raça especializadas como Holandês e Jersey. Ao determinar a composição do leite, é importante perceber que, dentro de um país, a composição não é constante. A composição do leite varia consideravelmente ao longo das estações (Lindmark-Månsson et al., 2003; Lock e Garnsworthy, 2003).

O estado do RS é responsável por $50 \%$ da produção total de leite e a região sudeste do Brasil por $42 \%$ da produção de alimentos lácteos (IBGE/PAM, 2008). O consumo médio per capita de produtos lácteos na região sul é o maior do país (IBGE, 2003). Devido aos possíveis efeitos benéficos à saúde observados com o consumo de CLA, associado à carência de evidências científicas informando a composição de ácidos graxos de leites desta região, o presente trabalho teve como objetivo principal comparar o teor de CLA em leites produzidos em diferentes regiões do Estado do Rio Grande do Sul durante as quatro estações do ano.

\section{Material e métodos}

Os produtos lácteos (leite UAT, queijo muçarela e manteiga) são as principais fontes alimentares de laticínios para a população que vive no Sul do Brasil (Nunes e Torres, 2010). Foram avaliadas 48 amostras de leites bovino fluido esterilizado (caixas de leite longa vida integral), de três diferentes laticínios, situados nas regiões do Noroeste, Norte e Sul do Rio Grande do Sul (RS), produzidos no ano de 2010, nas diferentes estações do ano, com quatro repetições e comercializadas na cidade de Santa Maria/RS. As análises foram realizadas no laboratório do Núcleo Integrado de Desenvolvimento em Análises Laboratoriais (NIDAL), Universidade Federal de Santa Maria, RS.

A extração de gordura dos leites foi realizada pelo método Bligh e Dyer (1959), com mistura de solvente água-metanol-clorofómio a frio. A preparação dos ésteres metílicos foi feita por meio do método proposto por Hartman e Lago (1973). O perfil de ácidos graxos e de CLA foi determinado por cromatografia gasosa (aparelho Agilent), utilizando-se uma coluna capilar de sílica fundida, SP-2560 (100m X 0,2mm X 0,2mm; Supelco) e detector de ionização de chama (FID). A duração da corrida foi de 70 minutos, a temperatura do injetor foi de $250^{\circ} \mathrm{C}$ e do detector de $300^{\circ} \mathrm{C}$. A injeção no modo "split", com relação $21: 1$. O gás de arraste foi o hidrogênio com fluxo de $40 \mathrm{ml} / \mathrm{minu}$ to e de pressão na cabeça da coluna.

A identificação dos picos foi efetuada pela comparação dos tempos de retenção com padrões de ésteres metílicos (FAMEs Mix, 37 components-Supelco) e como padrão interno sendo utilizado o ácido heptadecanoico. A quantificação foi determinada pela área do pico do éster metílico dos ácidos graxos, em relação à área total dos picos identificados, expresso em percentagem. Foram calculados a relação ácidos graxos saturados/ácidos graxos insaturados (AGS/AGI), soma de ômega-3 ( $\omega-3)$ e ômega-6 $(\omega-6)$ e a relação $\omega-6 / \omega-3$ e o teor de ácido linoleico conjugado (CLA).
Os resultados das análises serão submetidos à análise de variância (ANOVA) com blocos ao acaso com quatro repetições. A diferença foi analisada pelo teste $\mathrm{F}$ de Tukey, com nível crítico de significância a $5 \%$ de probabilidade, através do programa estatístico Statistical Analysis System (SAS) versão 9.3.1(SAS 2006).

\section{Resultados}

A Tabela 1 mostra o perfil de ácidos graxos das amostras de leites analisadas. Os 34 ácidos graxos listados constituem $100 \%$ do total de ácidos graxos, constituídos de ácidos graxos saturados e insaturados. Os teores de ácidos graxos saturados foram obtidos a partir da soma dos ácidos graxos saturados: butírico $(\mathrm{C} 4: 0)$, caproico (C6:0), enântico $(\mathrm{C} 7: 0)$, caprílico $(\mathrm{C} 8: 0)$, cáprico (C10:0), undecílico (C11:0), láurico (C12:0), mirístico (C14:0), pentadecílico (C15:0), palmítico (C16:0), margárico (C17:0), esteárico (C18:0), araquídico (C20:0), behênico (C22:0), tricosanóico (C23:0) e tetracoisanoico (C24:0). Os teores dos ácidos graxos monoinsaturados foram obtidos pela soma dos ácidos graxos monoinsaturados: miristoleico (C14:1), pentadecanoico (C15:1), palmitoleico (C16:1), heptadecanoico (C17:1), oleico (C18:1n9C), vacênico (C18:1n11t), elaídico (C20:1) e nervônico (C24:1). Já para o cálculo dos poliinsaturados, somou-se a concentração do linoleico (C18:2n6C), a-linolênico (C18:3n3), ácido linoleico conjugado (C18:2C9T11 e C18:2C12,T10), eicosadienoico (C20:2), di-homo-gamalinolênico (C20:3n6), araquidônico (C20:4n6), timnodônico (C20:5n3) e docosahexaenoico (C22:6n3).

Nunes e Torres (2012), em estudo com objetivo de desenvolver múltiplos modelos de regressão de isômeros de CLA em produtos lácteos, identificaram um total de 35 ácidos graxos, valores muito próximos a este estudo.

A composição de ácidos graxos (mg/100g de ácidos graxos totais) presente nos leites analisados permitiu identificação dos principais ácidos graxos saturados, rendendo um valor preciso de $67,71 \%$, sendo os mais abundantes o mirístico (14:0), o palmítico (16:0) e o esteárico (18:0). O total de ácidos graxos insaturados foi $32,51 \%$, o oleico $(18: 1 \mathrm{n} 9 \mathrm{c})$ foi o principal ácido graxo monoinsaturado nas amostras, contribuindo cerca de $23 \%$ do total de ácidos graxos. Assim como nesta pesquisa, Perfield et al. (2007) observaram maiores teores de ácidos graxos saturados o mirístico, o palmítico e o esteárico nas amostras de leite. Segundo Michalski e Januel (2006), os ácidos graxos saturados representam $60-70 \%$ de ácidos graxos total do leite e $30-35 \%$ de ácidos graxos insaturados (principalmente monoinsaturada). A composição média de ácidos graxos foi semelhante aos valores relatados por Jensen (2002).

Para a obtenção dos valores da relação de AGI/AGS, foi realizada a divisão dos valores dos ácidos graxos insaturados (AGI), soma dos monoinsaturados e os poliinsaturados, pelos ácidos graxos saturados (AGS), citados acima. A razão média entre insaturado e saturado foi aproximadamente de $0,48 \%$, valor muito próximo ao encontrado por Modesto (2002), equivalente a $0,51 \%$. Já Souza et al. (2004) observaram valores inferiores, $0,41 \%$. Segundo os padrões de referência, as recomendações do National Cholesterol Education Program (NEP) (1989) sugerem que a relação (AGS/AGI) deve ser inferior a $1,0 \%$, sendo seu principal objetivo a redução da morte por doença arterial coronariana e redução dos níveis de colesterol. 
Tabela 1: Composição de ácidos ( $\mathrm{mg} / 100 \mathrm{~g}$ de ácidos graxos totais) presente no leite

\begin{tabular}{|c|c|c|c|}
\hline$A G$ & Nomenclatura usual & $\begin{array}{c}\text { Média } \\
\mathrm{mg} / 100 \mathrm{~g}\end{array}$ & DP \\
\hline $4: 0$ & Ácido butírico & 0,99 & 0,35 \\
\hline $6: 0$ & Ácido caproico & 0,82 & 0,22 \\
\hline $8: 0$ & Ácido caprílico & 0,68 & 0,11 \\
\hline $10: 0$ & Ácido cáprico & 1,87 & 0,22 \\
\hline $11: 0$ & Ácido undecílico & 0,28 & 0,09 \\
\hline $12: 0$ & Ácido láurico & 2,57 & 0,10 \\
\hline $14: 0$ & Ácido mirístico & 10,59 & 0,43 \\
\hline $14: 1$ & Ácido miristoleico & 0,83 & 0,08 \\
\hline $15: 0$ & Ácido pentadecílico & 1,24 & 0,07 \\
\hline $15: 1$ & Ácido 10-pentadecenoico & 0,03 & 0,01 \\
\hline $16: 0$ & Ácido palmítico & 32,30 & 1,69 \\
\hline $16: 1$ & Ácido palmitoleico & 1,27 & 0,25 \\
\hline $17: 0$ & Ácido margárico & 0,92 & 0,04 \\
\hline $17: 1$ & Ácido 10-heptadecenoico & 0,31 & 0,43 \\
\hline 18:0 & Ácido esteárico & 14,92 & 0,89 \\
\hline $18: 1 n 9 t$ & Ácido elaídico & 0,56 & 0,26 \\
\hline $18: 1 \mathrm{n} 11 t$ & Ácido 7-octadecenoico & 2,95 & 0,28 \\
\hline $18: 1 n 9 c$ & Ácido oleico & 23,07 & 1,06 \\
\hline $18: 2 n 6 c$ & Ácido linoleico & 1,83 & 0,32 \\
\hline $20: 0$ & Ácido araquídico & 0,26 & 0,02 \\
\hline $20: 1$ & Ácido 5-eicosanoico & 0,00 & 0,00 \\
\hline $18: 3 n 3$ & Ácido alfa-linoleico & 0,31 & 0,22 \\
\hline $18: 2 \mathrm{n} 9 \mathrm{c} 11 t$ & Ácido linoleico cis-9, trans-11 & 0,72 & 0,25 \\
\hline $18: 2 \mathrm{n} 10 c 12 t$ & Ácido linoleico cis-10, trans-12 & 0,22 & 0,32 \\
\hline $20: 2$ & Ácido 8, 11-eicosadienoico & 0,03 & 0,03 \\
\hline $22: 0$ & Ácido behênico & 0,11 & 0,00 \\
\hline $20: 3 n 6$ & Ácido di-homo-gama-linoleico & 0,08 & 0,01 \\
\hline $20: 4 n 6$ & Ácido araquidônico & 0,12 & 0,03 \\
\hline 23:0 & Ácido tricosanoico & 0,09 & 0,01 \\
\hline $22: 2$ & Ácido 13, 16-docosadienoico & 0,03 & 0,02 \\
\hline $24: 0$ & Ácido lignocérico & 0,07 & 0,00 \\
\hline $20: 5 n 3$ & Ácido timnodônico & 0,10 & 0,03 \\
\hline $24: 1$ & Ácido nervônico & 0,03 & 0,01 \\
\hline $22: 6 n 3$ & Ácido cervônico & 0,02 & 0,01 \\
\hline
\end{tabular}

AG: Ácidos Graxos; DP: Desvio-Padrão

Os ácidos graxos (AG) são divididos em duas classes: os saturados e os insaturados. Os insaturados são divididos em monoinsaturados (MUFAS) e os poli-insaturados (PUFAS). Estes exercem vários efeitos potencialmente antiaterogênicos, incluindo redução dos níveis plasmáticos de LDL, aumento da vasodilatação e redução da agregação plaquetária (Kang e Leaf, 2000).

O teor de ômega-3 ( $\omega$-3) presente no leite foi maior na estação do inverno para a região Sul, com $0,75 \%$; a Noroeste não apresentou diferença nas estações da primavera e inverno, com média de $0,44 \%$ (Tabela 2). Os $\omega$-3 são uma classe essencial de ácidos graxos poli-insaturados. Estudos vêm examinando o papel dos ácidos graxos $\omega-3$ em relação a uma série de doenças, particularmente o câncer e as doenças cardiovasculares. $O$ aumento das evidências sugere que este ácido graxo apresenta um papel protetor nas doenças coronarianas e suas complicações, pois, metabolicamente, eles diminuem a produção hepática de triacilglicerol e a poliproteína $\mathrm{B}$, os principais constituintes lipídicos e proteicos das VLDL (lipoproteínas de densidade muito baixa) (Connor, 2000).

Tabela 2: Variação sazonal na composição de ácidos graxos $\omega 3$ no leite, pelo teste de Tukey

\begin{tabular}{cccccc}
\hline Local & Primavera & Verão & $\begin{array}{c}\text { Outono } \\
\mathrm{mg} / 100 \mathrm{~g}\end{array}$ & Inverno & Média \\
\hline Norte & $0,63 \mathrm{~b}$ & $0,66 \mathrm{ab}$ & $0,47 \mathrm{c}$ & $0,75 \mathrm{a}$ & 0.63 \\
Noroeste & $0,46 \mathrm{a}$ & $0,29 \mathrm{~b}$ & $0,29 \mathrm{~b}$ & $0,42 \mathrm{a}$ & 0,37 \\
Sul & $0,75 \mathrm{a}$ & $0,40 \mathrm{c}$ & $0,50 \mathrm{~b}$ & $0,57 \mathrm{~b}$ & 0,55 \\
Média & 0,61 & 0,45 & 0,42 & 0,58 & \\
\hline
\end{tabular}

Médias seguidas por letras minúsculas distintas na mesma linha, diferem entre estações $p<.0001$, pelo teste de Tukey.

O teor de ômega-6 presente no leite foi maior na estação verão para as regiões Norte e Noroeste, e a região Sul obteve maiores teores na primavera e inverno, $1,81 \%$ e $1,97 \%$, respectivamente (Tabela 3).

Tabela 3: Variação sazonal na composição de ácidos graxos $\omega 6$ no leite, pelo teste de Tukey

\begin{tabular}{cccccc}
\hline Local & Primavera & Verão & $\begin{array}{c}\text { Outono } \\
\mathrm{mg} / 100 \mathrm{~g}\end{array}$ & Inverno & Média \\
\hline Norte & $1,70 \mathrm{~b}$ & $3,31^{\mathrm{a}}$ & $1,74 \mathrm{~b}$ & $2,40 \mathrm{ab}$ & 2,29 \\
Noroeste & $1,86 \mathrm{a}$ & $2,33 \mathrm{Aa}$ & $1,83^{\mathrm{a}}$ & $2,15 \mathrm{a}$ & 1,95 \\
Sul & $1,81 \mathrm{a}$ & $1,62 \mathrm{~b}$ & $1,63 \mathrm{~b}$ & $1,97 \mathrm{a}$ & 1,76 \\
Média & 1,79 & 2,27 & 1,73 & 2,17 & \\
\hline
\end{tabular}

Médias seguidas por letras minúsculas distintas na mesma linha, diferem entre estações $p<.0001$ pelo teste de Tukey.

Os ácidos graxos mais importantes da família $\omega-6$ são os ácidos linoleico C18:2 (9,12), g-linolênico C18:3 $(9,12,15)$ e araquidônico C20:4 $(5,8,11,14)$. Os ácidos linoleico e araquidônico são ácidos graxos essenciais, ou seja, são indispensáveis ao organismo humano e, não sendo sintetizados pelo mesmo, devem ser ingeridos na dieta alimentar. Existem vários tipos diferentes de ácidos graxos ômega-6. A maioria é proveniente da dieta, como o ácido linoleico, por exemplo.

A razão entre a ingestão diária de alimentos fontes de ácidos graxos $\omega-6$ e $\omega-3$ assume grande importância na nutrição humana, resultando em recomendações que têm sido estabelecidas por autores e órgãos de saúde, em diferentes países (Martin et al., 2006). A razão $\omega-6 / \omega-3$ variou de 3:1 a 5:1 com relação às médias observadas neste estudo. Os valores relacionados evidenciam a tendência de convergência da razão entre os ácidos graxos $\omega-6$ e $\omega-3$ para o intervalo de 2:1 a 4:1. As razões de 2:1 a 3:1 têm sido recomendadas por alguns autores. Assim, as razões entre 2:1 e 4:1 têm maior importância para pessoas com hábitos alimentares que resultam em uma baixa ingestão de AEP (ácido eicosapentaenoico) e ADH (ácido docosahexaenóico). Por outro lado, dietas baseadas em razões $\omega-6 / \omega-3$ inferiores a 1:1 não são recomendadas, por inibirem a transformação do ácido linoleico em AGPI-CML (ácidos graxos de cadeia muito longa) (Martin et al., 2006). 
A necessidade de diminuir a razão $\omega-6 / \omega-3$ nas dietas modernas também tem sido sugerida pelos resultados de alguns estudos clínicos realizados na última década. Entre esses destacam-se: a diminuição de $70 \%$ na taxa de mortalidade em pacientes com doença cardiovascular, quando a razão AL/AAL (ácido linoleico/ alfa-linolênico) na dieta foi de $4: 1$; a redução nas inflamações decorrentes da artrite reumatoide, quando a razão $\omega-6 / \omega-3$ da dieta esteve entre $3: 1$ a $4: 1$, condição que foi alcançada pela suplementação com AEP, ADH e AAL; a diminuição dos sintomas decorrentes da asma, quando a razão $\omega-6 / \omega-3$ da dieta esteve ao redor de $5: 1$, sendo que em 10:1 os sintomas foram intensificados (Broughton et al., 1997).

As variações regionais e sazonais de níveis de CLA nos leites foram observadas em diferentes locais do Rio Grande do Sul. Os níveis de CLA no leite aumentaram nas estações da primavera e do inverno (Tabela 4) nas regiões estudadas, com exceção da região Sul, que não apresentou diferença significativa entre as estações, com média de $10,13 \mathrm{mg} / \mathrm{g}$ de gordura. Observando as diferenças entre os teores de CLA nas regiões, não houve diferença significativa entre as regiões na estação do inverno, com média de 9,84mg/g de gordura, sendo que a região Sul apresentou maior teor de CLA entre regiões, média de 10,13mg/g de gordura.

Tabela 4: Variação sazonal do ácido linoleico conjugado (CLA) no leite

\begin{tabular}{ccccc}
\hline \multirow{2}{*}{ Local } & Estação & Cis-9, trans-11 & $\begin{array}{c}\text { Trans-10, cis-12 } \\
\text { mg/g }\end{array}$ & Total de CLA \\
\hline \multirow{2}{*}{ Norte } & Primavera & $8,35 \mathrm{~B}^{\mathrm{a}} \pm 0,31$ & $0,67 \mathrm{~A}^{\mathrm{a}} \pm 0,09$ & $9,02 \mathrm{~B}^{\mathrm{a}} \pm 0,22$ \\
& Verão & $6,42 \mathrm{~B}^{\mathrm{b}} \pm 0,25$ & $0,65 \mathrm{~A}^{\mathrm{a}} \pm 0,05$ & $7,07 \mathrm{~B}^{\mathrm{b}} \pm 0,29$ \\
& Outono & $7,15 \mathrm{~B}^{\mathrm{b}} \pm 0,43$ & $0,70 \mathrm{~A}^{\mathrm{a}} \pm 0,08$ & $7,85 \mathrm{~B}^{\mathrm{b}} \pm 0,47$ \\
& Inverno & $8,80 \mathrm{~A}^{\mathrm{a}} \pm 0,46$ & $0,47 \mathrm{~B}^{\mathrm{b}} \pm 0,05$ & $9,27 \mathrm{~A}^{\mathrm{a}} \pm 0,45$ \\
\multirow{2}{*}{ Noroeste } & Primavera & $7,95 \mathrm{~B}^{\mathrm{a}} \pm 0,58$ & $0,52 \mathrm{~A}^{\mathrm{ab}} \pm 0,09$ & $8,47 \mathrm{~B}^{\mathrm{a}} \pm 0,60$ \\
& Verão & $5,73 \mathrm{~B}^{\mathrm{b}} \pm 0,64$ & $0,54 \mathrm{~A}^{\mathrm{a}} \pm 0,20$ & $6,27 \mathrm{~B}^{\mathrm{b}} \pm 0,66$ \\
& Outono & $5,13 \mathrm{C}^{\mathrm{b}} \pm 0,52$ & $0,53 \mathrm{~B}^{\mathrm{a}} \pm 0,03$ & $5,66 \mathrm{C}^{\mathrm{b}} \pm 0,49$ \\
& Inverno & $9,37 \mathrm{~A}^{\mathrm{a}} \pm 1,09$ & $0,30 \mathrm{~B}^{\mathrm{b}} \pm 0,0$ & $9,67 \mathrm{~A}^{\mathrm{a}} \pm 1,09$ \\
& Primavera & $9,92 \mathrm{~A}^{\mathrm{a}} \pm 0,89$ & $0,65 \mathrm{~A}^{\mathrm{a}} \pm 0,17$ & $10,57 \mathrm{~A}^{\mathrm{a}} \pm 1,05$ \\
& Verão & $8,80 \mathrm{~A}^{\mathrm{a}} \pm 0,40$ & $0,77 \mathrm{~A}^{\mathrm{a}} \pm 0,05$ & $9,57 \mathrm{~A}^{\mathrm{a}} \pm 0,41$ \\
& Outono & $9,35 \mathrm{~A}^{\mathrm{a}} \pm 0,46$ & $0,82 \mathrm{~A}^{\mathrm{a}} \pm 0,09$ & $10,17 \mathrm{~A}^{\mathrm{a}} \pm 0,38$ \\
& Inverno & $9,35 \mathrm{~A}^{\mathrm{a}} \pm 0,36$ & $0,87 \mathrm{~A}^{\mathrm{a}} \pm 0,20$ & $10,22 \mathrm{~A}^{\mathrm{a}} \pm 0,47$ \\
\hline
\end{tabular}

Médias seguidas por letras maiúsculas distintas na mesma coluna, diferem entre locais $p<.0001$ pelo teste de Tukey.

Médias seguidas por letras minúsculas distintas na mesma linha, diferem entre estações $p<.0001$ pelo teste de Tukey.

As principais fontes alimentares de CLA são o leite e produtos lácteos. O conteúdo específico de CLA no leite foi semelhante ao relatado por Martins et al. (2007), com média de 7,2mg/g de gordura. Dhiman et al. (1999) relataram que o conteúdo do CLA no leite variou de 7,3 a $9,0 \mathrm{mg} / \mathrm{g}$ de gordura, dependendo da dieta alimentar das vacas, sugerindo que pode ser aumentado significativamente, alterando a dieta. Nunes e Torres (2010) observaram valores médios de $7,47 \mathrm{mg} / \mathrm{g}$ de gordura em leites consumidos na região Sudeste do Brasil.

A gordura do leite caracteriza-se por ser uma das fontes naturais mais ricas de CLA (Collomb et al., 2006). A composição do leite pode variar de acordo com os seguintes fatores: raça, período de lactação, alimentação, saúde, idade, características individuais, clima, espaço entre as ordenhas e estação do ano (Venturini et al., 2007), onde a quantidade de CLA encontrada nos derivados de leites é reflexo direto da alimentação que foi oferecida aos animais.

A região, condições climáticas e períodos de lactação são conhecidos como mudanças sazonais que têm influências sobre a composição do leite. Especialmente, há uma negativa correlação entre a temperatura ambiente e da quantidade de gordura e proteína do leite (Ozrenk e Inci, 2008). Quando a temperatura aumenta, a quantidae de gordura e a proteína do leite diminuem. Ng-Kwaihang et al. (1984) e Lacroix et al. (1996) relataram que a percentagem de gordura foi infleunciada pela variação sazonal. Neste estudo a variação encontrada entre as amostras dentro de uma mesma estação do ano se deve às diferentes regiões de leites analisados.

O conteúdo de CLA em produtos lácteos pode variar muito, e os principais fatores que causam essa variação são relacionadas com os métodos da agricultura adaptadas e as dietas dos animais, especialmente quando ácidos graxos poli-insaturados são adicionados à dieta dos animais. O conteúdo de CLA também foi significativamente diferente entre as marcas comerciais para os tipos de leites analisados. As variações sazonais são reconhecidas como um fator que influencia o conteúdo CLA de produtos lácteos, que é maior durante o verão quando comparado ao inverno (Ledoux et al. 2005; Lock e Garnworthy, 2003). No presente estudo todos os produtos foram obtidos em diferentes épocas e regiões, e, portanto, variações na sua composição são esperadas. Essas variações podem ser decorrentes das dietas oferecidas aos animais, assim como o período de lactação.

As vacas podem apresentar grande variação individual nos níveis de CLA, Kelsey et al. (2003) encontram em sua pesquisa valores de CLA nos leite coletados no mesmo dia uma variação de até três vezes o conteúdo de CLA nos leites, apesar de todos os animais serem alimentados com a mesma dieta. Pode-se observar que nos resultados desta pesquisa houve variação nos teores de CLA entre produtos, que se deve a variação sazonal ou regional.

A nutrição pode ser considerada umas das mais importantes fontes de variação no rendimento e composição do leite, mas as condições climáticas e variação sazonal e diferenças regionais também podem desempenhar um papel importante. Também a qualidade e a composição do leite são da maior importância para a indústria de laticínios e para a saúde humana (Ozrenk e Inci, 2008).

\section{Conclusão}

O conteúdo CLA de leites produzidos na região Sul do Brasil possuem valores superiores aos relatados para os produtos lácteos de países em climas temperados. O isômero CLA mais abundante nos leites analisados foi o cis-9, trans- 11 .

A gordura do leite possui alguns ácidos graxos com propriedades biológicas de interesse para a saúde humana. Como demonstramos, o leite é uma excelente fonte de ácido linoleico conjugado, sendo que seus níveis variaram com as regiões e estações, onde apresentaram maiores teores na região sul do RS e nas estações da primavera e inverno. 


\section{Referências}

BLIGH, E. G.; DYER, W. J. A rapid method of total lipid extraction and purification. Can. Journal Physiology Pharmacology, v. 37, n. 8, p. 911-917, 1959.

BROUGHTON, K. S.; JOHNSON, C. S.; PACE, B. K.; LIEBMAN, M, KLEPPINGER KM. Reduced asthma symptoms with n-3 fatty acid ingestion are related to 5 -series leukotriene production. Canadian Journal Physiology Pharmacology, v. 65, n. 4, p. 10111017, 1997.

COLLOMB, M., BUTIKOFER, U., SIEBER, R., JEANGROS, B., BOSSET, J., 2002. Composition of fatty acids in cow's milk fat produced in the lowlands, mountains and highlands of Switzerland using high-resolution gas chromatography. International Dairy Journal 12, p. 649-659.

COLLOMB, M., SCHMID, A.; SIEBER, R.; WECHSLER, D.; RYHANEN, E. Conjugated linoleic acids milk fat: Variation and physiological effects. International Dairy Journal, v.16, n. 11, p.1347-1361, 2006.

CONNOR, W.E. Importance of $\mathrm{n}-3$ fatty acids in health and disease. American Journal Clinical Nutrition, v. 71, n.1, p.171S-5S, 2000.

DHIMAN, T. R.; SATTER, L. D.; PARIZA, M. W.; GALLI, M. P.; ALBRIGHT, K. Conjugated linoleic acid content of meat from steers fed soybean oil. Journal Dairy Science, v. 82, n. 1, p. 84, 1999.

EIFERT, E.C., LANA, R.P., LANNA, D.P.D., TEIXEIRA, M.A., ARCURI, P.B., LEAO, M.I., OLIVEIRA, M.V.M., VALADARES FILHO, S.C. Perfil de ácidos graxos e conteúdo de acido linoleico conjugado no leite de vacas alimentadas com a combinação de óleo de soja e fontes de carboidratos na dieta. Revista Brasileira de Zootecnia 35, p. 1829-1837, 2006.

HARTMAN, L.; LAGO, B. C. A rapid preparation of fatty methyl esters from lipids. Laboratory Practice, v. 22, n. 6, p. 475-477, 1973.

HECK, J.M.; VAN VALENBERG, H.J.; DIJKSTRA, J.; VAN HOOIJDONK, A.C. Seasonal variation in the Dutch bovine raw milk composition. Journal Dairy Science, v. 92, n. 10, p. 4745-4755, 2009.

HUR, S.J.; PARK, G.B.; JOO, S.T. Biological activities of conjugated linoleic acid (CLA) and effects of CLA on animal products. Livestock Science, v.110, n. 3, p. 221-229, 2007.

IBGE - Instituto Brasileiro de Geografia e Estatística, 2003. Aquisição alimentar domiciliar per capita anual, por Grandes Regiões, segundo os produtos - período 2002-2003 [Household food budget survey, annual per capita, by geographic regions, by product, years 2002-2003]. Retrieved October 29, 2007 from the IBGE [Brazilian Institute of Geography and Statistics] Home Page: http://www.ibge.gov.br/home/estatistica/populacao/ condicaodevida/

JENSEN, R.G. The composition of bovine milk lipids: January 1995 to December 2000. Journal Dairy Science, v. 85, n. 2., p. 295-350, 2002.

KELSEY, J.A.; CORL, B.A.; COLLIER, R.J.; BAUMAN, D.E. The effect of breed, parity, and stage of lactation on conjugated linoleic acid (CLA) in milk fat from dairy cows. Journal Dairy Science, v. 86, n. 8, p. 2588-2597, 2003.
LACROIX, C.; VERRET, P.; PAQUIN, P. Regional and seasonal variations of nitrogen fractions in commingled milk. International Dairy Journal, v. 6, p. 947-961, 1996.

LEDOUX, M.; CHARDIGNY, J. M.; DARBOIS, M.; SOUSTRE, Y.; SEBEDIO, J. L.; LALOUX, L. Fatty acid composition of French butters, with special emphasis on conjugated linoleic acid (CLA) isomers. Journal of Food Composition and Analysis, v.18, n. 5, p. 409-425, 2005.

LINDMARK-MÅNSSON, H.; FONDÉN, R.; PETTERSSON, H. E. Composition of Swedish dairy milk. International Dairy Journal, v.13, n. 6, p. 409-425, 2003.

LOCK, A. L.; GARNWORTHY, P. C. Seasonal variation in milk conjugated linoleic acid and D9-desaturase activity in dairy cows. Livestock Production Science, v. 79, n. 1, p. 47-59, 2003.

MARTINS, S.V.; LOPES, P.A.; ALFAIA, C.M.; RIBEIRO, V.S.; GUERREIRO, T.V.; FONTES, C.M.; CASTRO, M.F.; SOVERAL, G.; PRATES, J.A. Contents of conjugated linoleic acid isomers in ruminant-derived foods and estimation of their contribution to daily intake in Portugal. British Journal Nutrition, v. 98, n. 6, p.12061213, 2007.

MICHALSKI. M.C.; JANUEL. C. Does homogenization affect the human health properties of cow's milk? Trends in Food Science \& Technology, v.17, p.423-437, 2006.

MODESTO, E.C. Suplementação em pastagem de gramíneas tropicais com silagem do terço superior da rama de mandioca na alimentação de vacas leiteiras na época das águas. 2002. Tese (Doutorado) - Universidade Estadual de Maringá, Maringá, 2002.

NG-KWAI-HANG, K.F.; HAYES, J.F.; MOXLEY, J.E.; MONARDES, H.G.. Variability of test-day milk production and composition and relation of somatic cell counts with yield and compositional changes of bovine milk. Journal Dairy Science, v. 67, p. 361-366, 1984.

NUNES, J.C.; TORRES, A.G. Fatty acid and CLA composition of Brazilian dairy products, and contribution to daily intake of CLA. Journal of Food Composition and Analysis (Print), v. 23, p.782-789, 2010.

NUNES, J.C.; TORRES, A.G. Predicting conjugated linoleic acid (CLA) composition in Brazilian dairy products by multiple regression analysis based models. Dairy Science \& Technology, v. 92, p. 399413, 2012.

OZRENK, E.; INCI, S. S. The Effect of Seasonal Variation on the Composition of Cow Milk in Van Province Pakistan. Journal Nutrition, v.7, n.1, p.161-164, 2008.

PERFIELD, J. W. Trans-9, Cis-11 Conjugated Linoleic Acid Reduces Milk Fat Synthesis in Lactating Dairy Cows. Journal Dairy Science, n. 5, v. 90, p. 2211-2218, 2007.

STOOP, W. M.; VAN ARENDONK, J. A. M.; HECK, J. M. L.; VAN VALENBERG, H. J. F.; BOVENHUIS, H. Genetic parameters for major milk fatty acids and milk production traits of Dutch HolsteinFriesians. Journal Dairy Science, v. 91, n. 1, p. 385-394, 2008.

VENTURINI, K.S.; SARCINELLI, M.F.; SILVA, L.C. Características do leite. Boletim Técnico - PIE-UFES:01007 - Editado: 26/08/2007. Universidade Federal do Espírito Santo (UFES). Disponível em: http://www.agais.com/telomc/b1007 caracteristicas leite.pdf. Acesso em: 03 de dezembro de 2010. 